\title{
Body mass index is decreased in children and adolescents with postural tachycardia syndrome
}

\author{
Jing Lin ${ }^{1}$, Huacai Zhao ${ }^{2}$, Lei $\mathrm{Ma}^{3}$, Fuyong Jiao ${ }^{3}$ \\ ${ }^{1}$ School of Public Health; Departments of ${ }^{2}$ Urology and ${ }^{3}$ Pediatrics, the Third Affiliated Hospital of Medical College, Xi'an \\ Jiaotong University, Xi'an, China. E-mail: linjkaoyan2008@163.com
}

Received: 16th November 2017, Accepted: 24th December 2017

SUMMARY: Lin J, Zhao H, Ma L, Jiao F. Body mass index is decreased in children and adolescents with postural tachycardia syndrome. Turk J Pediatr 2019; 61: 52-58.

Our intent was to explore the predictive value of body mass index (BMI) in differentiating between vasovagal syncope (VVS) and postural tachycardia syndrome (POTS) in children and adolescents. A total of 111 children and adolescents with POTS and 154 children and adolescents with VVS were included in our study. The control group included 82 healthy children and adolescents. Height and weight were measured in all participants. The headup tilt test was performed in participants in all groups (POTS, VVS, and control). BMI was significantly lower in children and adolescents with POTS $(18.3 \pm 3.4)$ than in children and adolescents with VVS $(20.3 \pm 4.2)$ and the control group $(20.5 \pm 2.9)$. The receiver operating characteristic curve was performed to determine the predictive value of BMI differentiation between POTS and VVS and showed that a BMI of 19.30 was the cutoff value for the probability of distinction. However, the results (BMI of 19.30) produced unsatisfactory sensitivity $(\mathbf{5 7 . 1 \%})$ and specificity $(\mathbf{2 8 . 8 \% )}$ rates of correctly discriminating between patients with POTS and patients with VVS. Children and adolescents with POTS have a lower BMI compared with healthy peers or children and adolescents with VVS.

Key words: body mass index, postural tachycardia syndrome, vasovagal syncope, differential diagnosis.

Postural tachycardia syndrome (POTS) and vasovagal syncope (VVS) are main entities of orthostatic intolerance (OI) in children and adolescents. The mechanisms for these two types of OI are unclear, it is sometimes hard to distinguish between VVS and POTS. In the present study, we hypothesized that BMI levels might be different between children and adolescents with POTS and VVS, and that the BMI could be used to differentiate between patients with VVS and those with POTS. To our knowledge, we are the first to report on $\mathrm{BMI}$ in OI children and adolescents. In addition, we discuss the possible reasons why children and adolescents with POTS are thinner.

Orthostatic intolerance (OI) is a constellation of signs and symptoms that are elicited by standing upright and relieved by recumbency. ${ }^{1}$ OI symptoms include palpitations, light headedness, chest discomfort, shortness of breath, blurred vision, exercise intolerance, headache, nausea, abdominal pain, diminished concentration, tremulousness, syncope, near syncope, and hyperpnea. ${ }^{2,3}$ Postural tachycardia syndrome (POTS) and vasovagal syncope (VVS) are main entities of OI in children and adolescents. POTS is defined by symptoms of OI in association with excessive tachycardia ${ }^{2}$, whereas VVS is described by a sudden transient loss of consciousness and postural tone caused by cerebral hypoperfusion. ${ }^{4}$ POTS is more frequent in females and in most cases, occurs between the ages of 15 and 25 years old. Chronic fatigue, chronic headache, fibromyalgia, and sleep disturbances have been frequently associated with POTS, but VVS is most often infrequent and episodic. Patients with POTS may have sudden transient loss of consciousness, and patients with VVS may 
have chronic symptoms of OI. Because the mechanisms for these two types of OI are unclear, it is sometimes hard to distinguish between VVS and POTS merely by depending on the symptoms.

Christou and Kiortsis ${ }^{5}$ reviewed that, the lower the body mass index (BMI), the greater the predisposition to OI, accompanied by both the down-regulation of sympathetic nervous system activity and the up-regulation of parasympathetic nervous system activity. Stewart et al. ${ }^{6}$ found that the BMI in POTS patients with hypovolemia was significantly lower than in POTS patients with normal blood volume. Nevertheless, at present, there is no evidence determining whether the BMI associated with $\mathrm{OI}$ in children and adolescents could distinguish POTS from VVS. Therefore, in the present study, we hypothesized that BMI levels might be different between children and adolescents with POTS and VVS, and that BMI could be used to differentiate between patients with VVS and those with POTS.

\section{Material and Methods}

\section{Study design}

We conducted a retrospective study from June 2015 to May 2017; 265 children and adolescents who complained of OI were admitted to the Department of Pediatrics at Shaanxi Province People's Hospital. They were divided in POTS or VVS groups by using the head-up tilt test (HUTT). A total of 111 children and adolescents (48 boys and 63 girls) were diagnosed with POTS and 154 children and adolescents (71 boys and 83 girls) presented with VVS. Eightytwo (34 boys and 48 girls) children and adolescents served as controls. The control group was considered healthy based on medical history, physical examination and ECG. All children and adolescents were informed of the purposes of the research and agreed to provide relevant research information. Written informed consent was obtained from all study subjects/parents, the next of kin, or guardians of the minors/children enrolled in our study. The study was approved by the Xi'an Jiaotong University Ethics Committee in China. The study protocol conforms to the ethical guidelines of the 1975 Declaration of
Helsinki as reflected in a priori approval by the institution's human research committee.

\section{HUTT}

Children and adolescents were asked to stop using all medications that might affect autonomic function before the examination. The examination was performed in a quiet room at suitable room temperature equipped with medical resuscitation facilities. All children and adolescents were fasted at least four hours before the exam. Children lay on the tilt table (HUT-821; Beijing Juchi, Beijing, China) as their heart rate and blood pressure were consistently monitored by a Dash 2000 Multi-Lead Physiological Monitor (General Electric, NY, New York, U.S.A) until the heart rate stabilized. The table was tilted to a $60^{\circ}$ angle, and heart rate and blood pressure were monitored until either a positive response appeared or the exam (with a process of 45 minutes) was complete. ${ }^{7}$

\section{Measurement of weight and height}

Children and adolescents were asked to remove hats and shoes before measurement of height. Children and adolescents were measured with their backs against the wall and their head facing forwards. The measurer adjusted the slide plate to drop down slowly until there was contact with the cranial vertex by the bottom of the slide plate. The height was measured in centimeters to one decimal place. For measurement of weight, subjects wore only one layer of clothes. They stepped gently onto digital weighing scales, remaining in the upright position, and the reading was recorded (to one decimal place) after it became stable. BMI was calculated as the weight divided by the square of height.

\section{Diagnostic criteria of POTS and VVS}

POTS diagnostic criteria included (1) normal heart rate when supine and no evidence of any cardiovascular diseases; (2) $\geq 2$ of dizziness, headache, fatigue, blurred vision, chest tightness, palpitations, hand tremors, or syncope after standing; (3) increment heart rate $\geq 40$ beats $/ \mathrm{min}^{-1}$ or maximum heart rate $\geq 120$ beats $/ \mathrm{min}^{-1}$ after standing during the head-up tilt test, with at least 2 of the following symptoms: dizziness or vertigo, 
Table I. Baseline Characteristics of Study Participants.

\begin{tabular}{llllll}
\hline Characteristics & POTS & VVS & Control & F/ $2^{2}$ & P \\
\hline Number of cases & 111 & 154 & 82 & & \\
Gender (boy/girl) & $48 / 63$ & $71 / 83$ & $34 / 48$ & 0.515 & 0.773 \\
Age (years) & $11.6 \pm 2.8$ & $11.4 \pm 2.7$ & $10.7 \pm 2.3$ & 2.600 & 0.076 \\
Height (cm) & $150.2 \pm 15.8$ & $149.0 \pm 15.6$ & $145.3 \pm 12.8$ & 2.687 & 0.069 \\
Weight (kg) & $42.5 \pm 14.2$ & $45.7 \pm 13.9$ & $43.5 \pm 10.0$ & 2.023 & 0.134 \\
Supine HR (beats/min) & $73 \pm 10$ & $75 \pm 13$ & $85 \pm 12$ & 28.054 & $<0.001$ \\
Supine SBP (mmHg) & $101 \pm 8$ & $103 \pm 5$ & $109 \pm 11$ & 18.341 & $<0.001$ \\
Supine DBP (mmHg) & $57 \pm 11$ & $56 \pm 9$ & $64 \pm 10$ & 19.158 & $<0.001$ \\
HR at the end of HUTT (beats/min) & $116 \pm 16$ & $92 \pm 33$ & $104 \pm 12$ & 29.871 & $<0.001$ \\
SBP at the end of HUTT (mmHg) & $107 \pm 11$ & $87 \pm 18$ & $113 \pm 9$ & 107.216 & $<0.001$ \\
DBP at the end of HUTT (mmHg) & $66 \pm 10$ & $45 \pm 10$ & $71 \pm 7$ & 255.159 & $<0.001$ \\
\hline
\end{tabular}

POTS: postural tachycardia syndrome; VVS: vasovagal syncope; HR, heart rate; SBP, systolic blood pressure; DBP, diastolic blood pressure; HUTT: head-up tilt test

lightheadedness, headache, palpitations, complexion change, blurred vision, fatigue, discomfort in the morning, and syncope for severe cases; (4) symptoms relieved or diminished by recumbence and symptoms for $\geq 1$ month; and (5) exclusion of other cardiac, neurologic or metabolic diseases. Children and adolescents who met all the above-mentioned criteria were diagnosed with POTS. 8,9

VVS diagnostic criteria included (1) usually a school-aged child; (2) a history of syncope; (3) predisposing factors in most of the patients; (4) a positive response during HUTT; (5) exclusion of other cardiac, neurologic, or metabolic diseases. Positive responses during HUTT were as follows: children with syncope episode or presyncope with any of the following response during HUTT: (1) systolic blood pressure $\leq 80 \mathrm{mmHg}$ or diastolic blood pressure, $\leq 50 \mathrm{mmHg}$ or mean pressure decrease $\geq 25 \%$; (2) heart rate $<75$ beats/ min for children 4-6 years old; heart rate < 65 beats/min for children 7-8 years old; heart rate $<60$ beats/min for children older than 8 years; (3) electrocardiogram showed sinus arrest and premature junction contraction; (4) atrioventricular block and cardiac arrest $\geq 3$ seconds. ${ }^{10}$

\section{Statistical analysis}

The data were analyzed with SPSS 13.0 software. Enumeration data are described in the form of case number, and measurement data are described in the form of mean $\pm \mathrm{s}$. A $\chi^{2}$ test and variance test were used for the analysis of enumeration data and measurement data, respectively, among the three groups. The receiver operating characteristics (ROC) curve was used to explore the probability of correctly discriminating between patients with POTS and VVS by using the BMI. The area under the curve (AUC) was used to indicate the predictive value. An AUC from 0.5 to 0.7 indicated a lower predictive value; AUC from 0.7 to 0.9 indicated a moderate predictive value; AUC $>0.9$ indicated a high predictive value. $\mathrm{P}$ value $<0.05$ was considered statistically significant.

\section{Results}

\section{Baseline characteristics of study participants}

Of the 347 cases enrolled in the study, the participants had an average age of $11.3 \pm 2.6$ years, ranging from 6 to 18 years old. Boys constituted $44.1 \%$ of the sample $(n=153)$ and girls constituted $55.9 \%(n=194)$. A total of 111 children and adolescents met the diagnostic standard for POTS, constituting $32 \%$ of the participants. A total of 154 children and adolescents met the diagnostic standard for VVS accounting for $44.4 \%$ of the participants and the control group accounted for $23.6 \%$. There were no statistical differences among the POTS, VVS, and the control group in gender, 
Table II. Body Mass Index of Study Participants.

\begin{tabular}{llllll}
\hline Variables & POTS & VVS & Control & F & P \\
\hline Cases & 111 & 154 & 82 & & \\
Body mass index & $18.3 \pm 3.4^{* \#}$ & $20.3 \pm 4.2^{\&}$ & $20.5 \pm 2.9$ & 11.807 & $<0.001$ \\
\hline
\end{tabular}

POTS: postural tachycardia syndrome; VVS: vasovagal syncope

* $\mathrm{P}<0.001$ compared with control group

\# $\mathrm{P}<0.001$ compared with VVS group

$\& \mathrm{P}=0.933$ compared with control group

age, height, and weight. However, there were significant statistical differences in supine heart rate, supine systolic blood pressure, supine diastolic blood pressure, heart rate at the end of HUTT, systolic blood pressure at the end of HUTT, and diastolic blood pressure at the end of HUTT among the three groups (Table I).

\section{Body mass index of study participants.}

BMI was significantly lower in the POTS group compared with the VVS group and the control group. There was no statistical difference between BMIs of the VVS group compared with the control group (Table II).

Predictive value of BMI to differentiate between POTS and VVS

A ROC curve was used to detect the predictive value of BMI and showed that the AUC was 0.648 , with a $95 \%$ CI of 0.582 to 0.714 . A BMI of 19.30 was determined to be the cutoff value to differentiate between POTS and VVS, which produced a sensitivity of $57.1 \%$ and a specificity of $28.8 \%$ (Fig. 1).

\section{Discussion}

The present study showed that BMI was significantly lower in POTS patients compared with VVS patients or healthy controls. Although the predictive value of the BMI for discriminating between patients with VVS and those with POTS was low, it is acceptable. According to this study, when the BMI of a patient is $>19.30$, the patient may have VVS.

\section{Difficulty in identify POTS and VVS}

The current diagnosis of OI is mainly based on age, predisposing factors, clinical manifestations, and HUTT; however, it is difficult to identify VVS and POTS only by relying on these characteristics. First, it is impossible to differentiate the two subtypes from the clinical manifestation since patients with POTS may have sudden transient loss of consciousness and patients with VVS may have chronic symptoms of OI. Second, the HUTT cannot differentiate POTS from VVS because in some cases orthostasis that occurs in VVS is accompanied by large increases in heart rate (HR) that should not be construed as POTS. ${ }^{11}$ Third, there are no special differences between the two types in terms of age and predisposing factors.

\section{Different in treatment of POTS and VVS}

The hemodynamic mechanisms and treatment

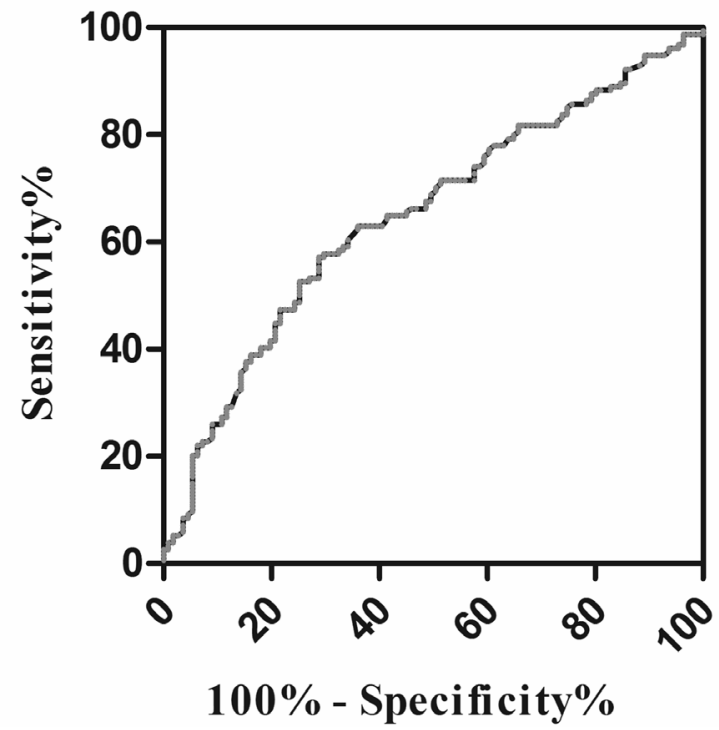

Fig. 1. The receiver-operating characteristic (ROC) curve for BMI for predicting the differential diagnosis between vasovagal syncope (VVS) and postural orthostatic tachycardia syndrome (POTS) in children and adolescents. The $y$-axis represents the sensitivity of predicting the effectiveness of BMI levels between patients diagnosed with VVS or POTS. The x-axis represents the false-positive rate (1-specificity) of the prediction. The area under the curve was $0.648(\mathrm{P}<0.01)$ 
regimens for POTS and VVS are different. Currently, therapy for POTS is similar to the treatment for neurogenic orthostatic hypotension in the use of physical countermeasures, salt and water intake, and pharmacotherapy. ${ }^{12}$ Exercise has always been a mainstay of rehabilitation in these patients. Recent work indicates that gravitational deconditioning (e.g., bedrest) is a frequent concomitant of the illness and that a graded exercise program can be very effective in improving overall patient wellbeing. ${ }^{13}$ Therapy for VVS that is associated with a lengthy prodrome is largely avoidance and physical countermeasures; the most efficacious of these is to lie down or squat. Other countermeasures include those that enhance the skeletal muscle pump (e.g., leg crossing) or activate the exercise pressor reflex (isometric hand grip). Enhanced salt and water intake is often encouraged and has shown some efficacy in small studies employing large amounts of salt loading. ${ }^{12,14}$ Moreover, the pathogenesis for POTS is mainly abnormal vasodilatation and central hypovolemia, hence, the $\alpha 1$-adrenergic agonist midodrine and the supplementary use of oral rehydration salts is used to cope with the syndrome. An abnormal Bezold-Josch reflex is the main cause for VVS, and $\beta$-blockers, such as metoprolol, are used. Hence, it is very important to distinguish the two subtypes.

\section{A small number of reports on differential diagnosis and their limitations}

Only a few studies have reported on the differentiation between POTS and VVS. Zhang et al. ${ }^{7}$ found that plasma hydrogen sulfide $\left(\mathrm{H}_{2} \mathrm{~S}\right)$ can be used in differentiating between VVS and POTS in children. The results showed that plasma levels of $\mathrm{H}_{2} \mathrm{~S}$ were significantly higher in children with VVS $(95.3 \pm 3.8 \mu \mathrm{mol} / \mathrm{L})$ and POTS $(100.9 \pm 2.1 \mu \mathrm{mol} / \mathrm{L})$ compared with children in the control group $(82.6 \pm 6.5 \mu \mathrm{mol} / \mathrm{L})$. Compared with the VVS group, the POTS group demonstrated significantly increased $\mathrm{H}_{2} \mathrm{~S}$ plasma levels ${ }^{7}$. However, the electrode senses sulfide, but not necessarily direct $\mathrm{H}_{2} \mathrm{~S}$. $\mathrm{H}_{2} \mathrm{~S}$ detection relies on its conversion to ionized sulfide in a sulfide antioxidant buffer, which, in blood or plasma, also receives very large sulfide contributions from desulfurated cysteines rather than dissolved $\mathrm{H}_{2} \mathrm{~S}$. Directly mixing blood and the sulfide antioxidant buffer greatly overestimates free sulfide. Moreover, $\mathrm{H}_{2} \mathrm{~S}$ is not specific to these diseases, and there are other diseases, such as chronic obstructive lung disease, coronary heart disease, hypertension, pulmonary hypertension, and type- 1 and -2 diabetes mellitus, in which $\mathrm{H}_{2} \mathrm{~S}$ is altered. Li et al. ${ }^{15}$ found that serum iron might be used as an initial diagnostic method to differentiate between VVS and POTS. The results revealed that there was a significant difference between the median serum iron value of POTS [17.4 (interquartile range 13.5 -21.8) $\mu \mathrm{mol} / \mathrm{L}$ ] and VVS [8.9 (interquartile range $7.5-17.6) \mu \mathrm{mol} / \mathrm{L}] .{ }^{15}$ However, only 40 POTS patients and 17 VVS patients were enrolled in the study, therefore, the sample is not representative and the results need further validation. Therefore, it is meaningful to identify better indicators to differentiate POTS from VVS.

\section{BMI was reduced in patients with decreased peripheral blood flow}

A previous study was conducted that included 52 patients with POTS (14-29 years old) compared with 36 control subjects (14-27 years old). 6 The results showed that BMI was not significantly reduced in the POTS patients, but was reduced in patients with decreased peripheral blood flow ${ }^{6}$. Patients with low-flow POTS had decreased body mass. Nevertheless, the participants in this study were not exclusively children or adolescents; therefore, a study determining whether BMI could be used to differentiate between POTS and VVS in children and adolescents was necessary.

\section{POTS patients have high AngII which is inversely correlated with BMI}

As was expected, in the present study, the BMI in children and adolescents with POTS was significantly lower than in VVS and healthy subjects. Why is BMI reduced in POTS subjects? Stewart discovered that low-flow POTS patients have abnormally high AngII and, in these patients, AngII is inversely correlated with BMI. ${ }^{6}$ Goossenset al. ${ }^{16}$ demonstrated that lipolysis could be inhibited by AngII in adipose and skeletal muscle tissue in both obese and normal-weight subjects using microdialysis techniques. Porter et al. ${ }^{17}$ suggested that in adult rats AngII acts in the brain to affect food intake and energy expenditures in a manner 
that is not related to water intake. Previous studies have demonstrated the potential for increased AngII within the CNS to produce sympatho-excitation, which is a major factor in hyperadrenergic POTS. Hence, we assume that AngII may be correlated with POTS or VVS children and adolescents, and future work will focus closely on such possibilities.

Gene, leptin and ghrelin that are related with blood pressure and heart rate may be involved in different orthostatic responses between individuals with different BMI values.

A previous study suggested that a genetically determined joint regulation of BMI and orthostatic responses occurs by the same gene. ${ }^{18}$ This finding suggests that a gene(s) on chromosome $13 \mathrm{q}$ jointly regulates the systolic blood pressure (SBP) response to postural change and BMI. ${ }^{18}$ Some individuals who are constitutionally thin are possibly genetically predisposed to experience OI, whereas adults with a chronic history of obesity from childhood and usually with a relevant family history of obesity may be genetically protected against OI. Geloneze et al. ${ }^{19}$ found a strong link between leptin and BMI, and that BMI is positively correlated with serum leptin levels. There is increasing evidence that leptin, not only increases satiety, but also can up-regulate sympathetic nervous system activity and tends to increase BP and HR. ${ }^{20-}$ 22 Whereas, ghrelin administration stimulates growth hormone secretion, it also causes weight gain by increasing food intake and reducing fat utilization in rodents. ${ }^{23}$ Ghrelin is secreted by the stomach to increase appetite; it has been found to down-regulate sympathetic nervous system activity and inhibit endothelin1-induced vasoconstriction, both leading to vasodilation with reduction of blood pressure ${ }^{23}$. We postulate that leptin and ghrelin may be involved in differential orthostatic responses between individuals with different BMI values.

\section{Low predictive value but acceptable}

Although there are many theories to explain the possibility that BMI correlates with OI or sympathetic nervous system, the evidence remains unclear. Thus, why children and adolescents with POTS are much thinner needs further exploration. BMI has a low predictive value for the differentiation between VVS and POTS; however, it is acceptable, because there is not a parameter that can be used alone for differential diagnosis. Illness recognition should depend on medical history, clinical criteria, laboratory inspection, and other new methods of identification.

\section{Study limitations}

There are limitations to the current study. It is a single-centered study, and a multi-center research study is needed in the future. However, the present study discovered for the first time that BMI could be used as a useful index to differentiate patients with POTS from those with VVS. Although the predictive value of BMI in the differential diagnosis between POTS and VVS was not satisfactory, it is still acceptable. In addition, to our knowledge, we are the first to report on BMI in OI children and adolescents.

\section{Funding}

This work was supported by the National Natural Science Foundation of China [grant number 81803263 to JL].

\section{REFERENCES}

1. Low PA, Sandroni P, Joyner M, Shen WK. Postural tachycardia syndrome (POTS). J Cardiovasc Electrophysiol 2009; 20: 352-358.

2. Jarjour IT. Postural tachycardia syndrome in children and adolescents. Semin Pediatr Neurol 2013; 20: 1826.

3. Stewart JM. Common syndromes of orthostatic intolerance. Pediatrics 2013; 131: 968-980.

4. Folino AF, Russo G, Porta A, Buja G, Cerutti S, Iliceto $\mathrm{S}$. Modulations of autonomic activity leading to tiltmediated syncope. Int J Cardiol 2007; 120: 102-107.

5. Christou GA, Kiortsis DN. The effects of body weight status on orthostatic intolerance and predisposition to noncardiac syncope. Obes Rev 2017; 18: 370-379.

6. Stewart JM, Taneja I, Medow MS. Reduced body mass index is associated with increased angiotensin II in young women with postural tachycardia syndrome. Clin Sci (Lond) 2007; 113: 449-457.

7. Zhang F, Li X, Stella C, et al. Plasma hydrogen sulfide in differential diagnosis between vasovagal syncope and postural orthostatic tachycardia syndrome in children. J Pediatr 2012; 160: 227-231.

8. Li H, Han Z, Chen S, et al. Total peripheral vascular resistance, cardiac output, and plasma C-type natriuretic Peptide level in children with postural tachycardia syndrome. J Pediatr 2015; 166: 1385-1389.e1-2.

9. Zhao J, Du S, Yang J, et al. Usefulness of plasma copeptin as a biomarker to predict the therapeutic effectiveness of metoprolol for postural tachycardia syndrome in children. Am J Cardiol 2014; 114: 601605. 
10. Lin J, Wang Y, Ochs T, Tang C, Du J, Jin H. Tilt angles and positive response of head-up tilt test in children with orthostatic intolerance. Cardiol Young 2015; 25: 76-80.

11. Medow MS, Merchant S, Suggs M, Terilli C, O’DonnellSmith B, Stewart JM. Postural heart rate changes in young patients with vasovagal syncope. Pediatrics 2017; 139: pii: e20163189.

12. Stewart JM. Mechanisms of sympathetic regulation in orthostatic intolerance. J Appl Physiol (1985) 2012; 113: 1659-1668.

13. Furlan R, Jacob G, Snell M, et al. Chronic orthostatic intolerance: A disorder with discordant cardiac and vascular sympathetic control. Circulation 1998; 98 : 2154-2159.

14. Claydon VE, Hainsworth R. Salt supplementation improves orthostatic cerebral and peripheral vascular control in patients with syncope. Hypertension 2004; 43: 809-813.

15. Li JW, Zhang QY, Gao J, Jin HF, Du JB. Significance of serum iron in the differential diagnosis between vasovagal syncope and postural orthostatic tachycardia syndrome in children. Beijing Da Xue Xue Bao Yi Xue Ban_2013; 45: 923-927.

16. Goossens GH, Blaak EE, Saris WH, van Baak MA. Angiotensin II-induced effects on adipose and skeletal muscle tissue blood flow and lipolysis innormal-weight and obese subjects. J Clin Endocrinol Metab 2004; 89: 2690-2696.
17. Porter JP, Potratz KR. Effect of intracerebroventricular angiotensin II on body weight and food intake in adult rats. Am J Physiol Regul Integr Comp Physiol 2004; 287: R422-R428.

18. North KE, Rose KM, Borecki IB, et al. Evidence for a gene on chromosome 13 influencing postural systolic blood pressure change and body mass index. Hypertension 2004 ;43: 780-784.

19. Geloneze B, Tambascia MA, Pareja JC, Repetto EM, Magna LA, Pereira SG. Serum leptin levels after bariatric surgery across a range of glucose tolerance from normal to diabetes. Obes Surg 2001; 11: 693-698.

20. Dunbar JC, Hu Y, Lu H. Intracerebroventricular leptin increases lumbar and renal sympathetic nerve activity and blood pressure in normal rats. Diabetes 1997; 46: 2040-2043.

21. Paolisso G, Manzella D, Montano N, Gambardella A, Varricchio M. Plasma leptin concentrations and cardiac autonomic nervous system in healthy subjects with different body weights. J Clin Endocrinol Metab 2000; 85: $1810-1814$.

22. Frühbeck G. Pivotal role of nitric oxide in the control of blood pressure after leptin administration. Diabetes 1999; 48: 903-908.

23. Tschöp M, Weyer C, Tataranni PA, Devanarayan V, Ravussin E, Heiman ML. Circulating ghrelin levels are decreased in human obesity. Diabetes 2001; 50: 707-709. 